\title{
Propofol versus placebo (with rescue with ketamine) before less invasive surfactant administration: study protocol for a multicenter, double-blind, placebo controlled trial (PROLISA)
}

\author{
Marie Chevallier ${ }^{1,2^{*}}$, Xavier Durrmeyer ${ }^{3,4,5}$, Anne Ego $^{2}$, Thierry Debillon ${ }^{1,2}$ and The PROLISA Study Group
}

\begin{abstract}
Background: One major limitation for less invasive surfactant administration (LISA) is the difficulty in providing sedation before this procedure and the competitive risk of respiratory depression versus avoidance of intubation for most sedative or analgesic drugs used in this context.

The objective of this study is to compare the need for mechanical ventilation within $72 \mathrm{~h}$ of life following premedication with propofol, versus placebo (rescue with ketamine), for the LISA procedure in preterm neonates born before 32 weeks gestational age (wGA).

Methods: ProLISA is a phase III, non-inferiority, multicenter, double blind, randomized, placebo controlled trial designed according to the SPIRIT Statement. Neonates born before 32 wGA in 12 geographically dispersed Neonatal Intensive Care Units in France needing surfactant will be included from September 2019 to September 2022. A sample of 542 patients is needed. The neonate is randomized to the intervention (propofol) or control placebo group. Open label rescue treatment with ketamine is possible in both groups if FANS (Faceless Acute Neonatal pain Scale) is $\geq 6$. To guide drug administration, FANS is scored before attempting laryngoscopy. Once an adequate score has been obtained, LISA is performed according to a standardized protocol. The primary outcome is the need for mechanical ventilation within $72 \mathrm{~h}$ of life. Secondary outcomes are tolerance of the procedure, pain evaluation, hemodynamic and neurologic parameters after the intervention, morbidities before discharge and neurodevelopmental assessment at 2 years of age.
\end{abstract}

Discussion: This paper describes the first multicenter, double-blind, randomized, placebo-controlled trial on this topic and will provide crucial information to support implementation of the LISA procedure.

Trial registration: ClinicalTrials.gov: NCT04016246. Registered 06 June 2019, NEUDRACT: 2018-002876-41.

Keywords: Less invasive surfactant administration, Sedation, Propofol, Ketamine, Randomized study

\footnotetext{
* Correspondence: mchevallier3@chu-grenoble.fr

'UMR 5525 ThEMAS, CNRS, TIMC-IMAG, Grenoble Alps University, Grenoble, France

${ }^{2}$ Neonatal Intensive Care Unit, Grenoble Alps University Hospital, Grenoble, France

Full list of author information is available at the end of the article
}

C C The Author(s). 2020 Open Access This article is licensed under a Creative Commons Attribution 4.0 International License, which permits use, sharing, adaptation, distribution and reproduction in any medium or format, as long as you give appropriate credit to the original author(s) and the source, provide a link to the Creative Commons licence, and indicate if changes were made. The images or other third party material in this article are included in the article's Creative Commons licence, unless indicated otherwise in a credit line to the material. If material is not included in the article's Creative Commons licence and your intended use is not permitted by statutory regulation or exceeds the permitted use, you will need to obtain permission directly from the copyright holder. To view a copy of this licence, visit http://creativecommons.org/licenses/by/4.0/. The Creative Commons Public Domain Dedication waiver (http://creativecommons.org/publicdomain/zero/1.0/) applies to the data made available in this article, unless otherwise stated in a credit line to the data. 


\section{Background}

Respiratory Distress Syndrome (RDS) affects $85 \%$ of preterm babies born before 32 weeks gestational age (wGA). Currently, the strategy to manage RDS in neonates who do not require intubation in the delivery room includes non-invasive nasal ventilation, early rescue treatment with animal derived surfactant, and the limitation of mechanical ventilation (MV). In fact, $\mathrm{MV}$ is one of the main risk factors of bronchopulmonary dysplasia (BPD) because it leads to chronic inflammation [1-3]. A reduction in the duration of $\mathrm{MV}$ is also one of the ways to limit the risk of $\mathrm{BPD}$ [4]. European guidelines from 2019 state that "LISA [less invasive surfactant administration] is the preferred mode of surfactant administration for spontaneously breathing babies on continuous positive airway pressure (CPAP), provided that clinicians are experienced with this technique" [5]. In the LISA procedure, the operator inserts a thin tube to administer intra-tracheal surfactant to spontaneously breathing preterm infants receiving non-invasive ventilation [615]. After surfactant administration, the catheter is immediately removed and non-invasive ventilation is continued [6]. The main advantage of this strategy is to avoid ventilator-induced lung injury. Two metaanalyses reported a $30 \%$ decrease in MV within the first $72 \mathrm{~h}$ and in bronchopulmonary dysplasia (BPD) at 36 weeks with the use of LISA [16, 17]. These meta-analyses pooled and studied the data of several randomized trials about LISA versus other strategies. One of the barriers to the wider implementation of the technique is the absence of consensus on premedication [18]. In fact, most studies were performed without sedation or with sedation at the discretion of the clinicians [19].

Thus an ideal drug for this context should have fast onset, short action, rapid offset, provide good sedation and analgesia with the least possible adverse effects and especially little impact on respiratory drive.

Currently there is no international and national consensus concerning specific premedication prior to the LISA procedure. Unfortunately the most interesting drugs induce respiratory depression and could lead to intubation, which is exactly what the LISA procedure tries to avoid. Propofol, ketamine and fentanyl have been reported in the literature as premedication for the LISA procedure $[11,13,14,20]$. In existing studies using premedication, the overall population had longer gestational ages but slightly higher MV rates as compared to studies without premedication.

Propofol might be of interest due to its short action, quick preparation, rapid onset and sedative properties [21]. It has been shown to be a suitable sedative for nonemergent neonatal endotracheal intubation with good tolerance and the possibility of titration [22, 23]. An observational study reported a higher level of comfort (ComfortNeo scale) when using premedication with 1 $\mathrm{mg} / \mathrm{kg}$ propofol for the LISA procedure compared to a historical non-sedated control group [11]. An unmasked, single-center randomized, controlled trial of $1 \mathrm{mg} / \mathrm{kg}$ propofol versus no premedication during LISA found a significant decrease in the pain score (ComfortNeo) but more frequent desaturations in the propofol group. No significant difference was observed for the rate of intubation, but the study might have been underpowered for this outcome [20].

Ketamine has rapid onset, a short duration of action, good analgesic and amnesic properties and maintains cardiovascular and respiratory stability [24]. An observational study reported the use of ketamine titrated by 0.5 $\mathrm{mg} / \mathrm{kg}$ increments in neonates prior to the LISA procedure [13]. The intubation rate was $24 \%$ during the procedure and $41 \%$ within $72 \mathrm{~h}$, underlining the difficulty to assess the depth of sedation. However, the majority of infants had faceless acute neonatal pain scores (FANS) below the threshold for pain. In another observational study on neonatal intubation in the delivery room, ketamine was associated with decreased pain scores, as compared to no premedication [25].

Briefly, propofol and ketamine seem to be effective for reducing pain scores, with acceptable tolerance for the LISA procedure, although propofol seems to be easier to titrate in this population.

Since 1990, studies have demonstrated that direct laryngoscopy is a painful and stressful procedure [26] that is why performed LISA without premedication is hard to discuss. It may cause bradycardia and intracranial hypertension when it is performed without sedation [27, 28]. For this reason, several academic bodies have recommended the use of premedication before neonatal intubation, except in life-threatening situations [26, 29]. Despite this evidence, a Scandinavian survey showed that only half of responding NICUs always used premedication before a LISA procedure, although it requires a laryngoscopy similar to that required by endotracheal intubation [30]. The clinicians' major concern about using premedication before LISA is the opposing goals of obtaining efficient sedation/analgesia while maintaining respiratory drive. For these reasons, this study assesses the impact of sedation during LISA on the rate of MV. Our hypothesis is that using premedication with propofol in a standardized protocol will not dramatically increase the need for MV within $72 \mathrm{~h}$.

The objective is to conduct a non-inferiority study to compare the need for MV within $72 \mathrm{~h}$ of life following premedication with propofol or placebo before the LISA procedure in preterm babies $<32 \mathrm{wGA}$. 


\section{Methods/design}

\section{Study setting and design}

PROLISA is a phase III, non-inferiority, multicenter, double blind, randomized, controlled trial comparing propofol vs placebo. The trial involves 12 geographically dispersed NICUs in France. These neonatal units regularly participate in health research and have a varied case mix of infants. All centers already use the LISA procedure as a standard of care. In each center, a PROLISA referent neonatologist has been appointed.

\section{Study sample and recruitment}

Infants born before 32 wGA are included as soon as possible after birth from September 2019 to September 2022, once they meet the inclusion criteria:

- presenting a RDS in the first $48 \mathrm{~h}$ of life and treated by non-invasive ventilation requiring surfactant with the following $\mathrm{FiO}_{2}$ to obtain a pulse oxymetry $\left(\mathrm{SpO}_{2}\right)$ between 88 and $95 \%$

- $\mathrm{FiO}_{2} \geq 30 \%$ for a duration $\geq 10$ min for infants born between 28 and 31 wGA

- $\mathrm{FIO}_{2} \geq 25 \%$ for a duration $\geq 10$ min for infants born $<28$ wGA;

- available intravenous line (peripheral, umbilical or central catheter);

- covered by the French Social Security;

- signed parental informed consent.

Non-inclusion criteria are:

- congenital and/or major malformations, including upper airway malformations;

- $\mathrm{FiO}_{2}>60 \%$ at the time of inclusion;

- Silverman score $>6$;

- Contra-indication to the use of propofol:

- low mean arterial blood pressure at 2 successive measurements (< gestational age expressed in weeks persisting after one volume expansion),

- use of inotropic medication to maintain a normal blood pressure.

- use of sedative or analgesic drugs (except paracetamol and ibuprofen) in the previous $24 \mathrm{~h}$

- coma, seizures, areactivity at neurological examination

\section{Interventions}

\section{Management before procedure}

After verifying the neonate's eligibility, obtaining the mother's, and ideally both parents' consent, the newborn is randomized to the Propofol or placebo arm. For the intervention, the newborn is prepared as per usual practice for tracheal intubation, whether admitted to the $\mathrm{NICU}$ or resuscitated in the delivery room, equipped with cardio-respiratory monitors, $\mathrm{SpO}_{2}$ monitoring (sensor placed on right hand). The equipment for the LISA procedure and for tracheal intubation (endotracheal tube, laryngoscope and Magill Forceps) is prepared. Non-invasive respiratory support (with devices routinely used in the centre) is maintained throughout the procedure using Non Invasive Positive Pressure Ventilation (NIPPV) or CPAP with Positive End Expiratory Pressure (PEEP) $\geq 4 \mathrm{~cm} \mathrm{H}_{2} 0$, Positive Inspiratory Pressure (PIP) $\geq 6 \mathrm{~cm} \mathrm{H}_{2} \mathrm{O}$, and respiratory frequency at the discretion of the clinicians. However, we propose a respiratory rate around 30/ min. NIPPV devices must have including variable flow and constant flow generators.

In the two groups, when the intervention team is ready, a solution of atropine, caffeine and oral sugar solution $30 \%$ or $24 \%$ is given drop, wise orally using a syringe before the LISA procedure. The posology of drugs are chosen according to the protocol routinely in use locally (around $10 \mu \mathrm{g} / \mathrm{kg}$ for atropine, $20 \mathrm{mg} / \mathrm{kg}$ for caffeine and 5-10 drops for sucrose). If LISA is performed in delivery room, caffeine should be given at soon as possible after procedure. It is common practice in France for atropine to be used before any intubation procedure. The aim of the atropine administration is to reduce the risk of bradycardia associated with an exacerbation of vagal tone. Sucrose is given as an analgesic agent [31] and caffeine is used to avoid central apnea [32].

\section{Intervention: sedation in both experimental and control groups}

The administration of propofol (experimental group) or placebo (control group) is performed by titration: (0.5 $\mathrm{mg} / \mathrm{kg}$ per dose of propofol or a similar volume of placebo (20\% Medialipide).

Within 2 min of injection of the first dose, a FANS pain score is rapidly evaluated after firmly rubbing the neonate's heel; according to a previously published intubation readiness score [33] (Fig. 1): The FANS scale (/10) allows a painful procedure to be evaluated, whether the face is visible or not.

- If the FANS score is $\geq 6$, a second dose of $0.5 \mathrm{mg} / \mathrm{kg}$ is injected. After two (<28 wGA) or $3(28-31 \mathrm{wGA})$ administrations of the drug (or placebo), if sufficient comfort is not achieved (FANS $\geq 6$ ) an open label rescue treatment is given: ketamine at $0.5 \mathrm{mg} / \mathrm{kg}$. One or two injections are recommended, but in some cases further doses of ketamine doses might be needed according to the discretion of the clinician. The latter should be an exceptional case, and 1 or 2 doses should be sufficient in most cases. This open-label treatment with ketamine is 


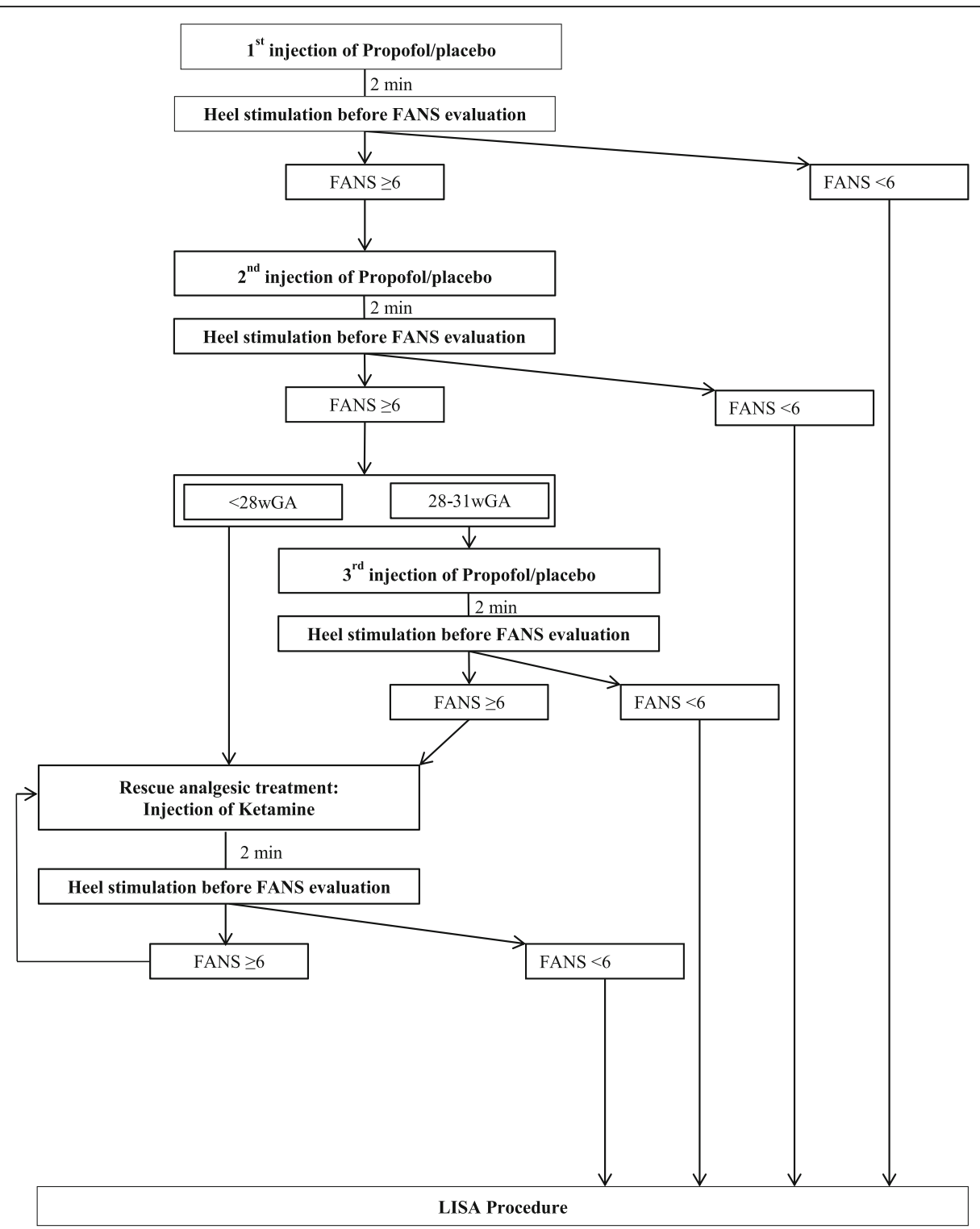

Fig. 1 Intervention scheme

consistent with expert recommendations based on trials evaluating pain treatment in neonates [34].

- If the FANS score is $<6$, no further injection of propofol (or placebo) is given.

\section{The LISA procedure}

An aspiration probe $(\mathrm{CH} 6)$ or the LISAcath ${ }^{\oplus}$ (Catheter for oral endotracheal instillation, CHIESI SAS, Bois Colombes, France) is used. The type of probe is left to the choice of each investigator. If an aspiration probe is used, it is marked to indicate the desired depth of insertion $(6 \mathrm{~cm}+$ weight if the procedure is performed through the nose, $7 \mathrm{~cm}+$ weight if the procedure is performed through the mouth with a nasal mask left in place during the procedure). Direct laryngoscopy or indirect laryngoscopy (with videolaryngoscopy), according to local practice, is performed with the probe inserted to beyond the vocal cords at the required depth, and held in position at the lips. Once the probe is correctly positioned, surfactant is slowly infused at a dose specified by the local protocol. At the end of the administration, the probe is immediately removed. The newborn continuously remains on NIPPV throughout the procedure, since in previous trials using propofol for LISA, NIPPV was found to be required continuously $[11,20]$.

If the newborn develops transient apnoea, inflations providing additional positive pressure are given using the respiratory support (NIPPV), an $\mathrm{Ambu}^{\oplus}$ bag or a Tpiece resuscitator device (Neopuff ${ }^{\circledR}$. 
The previous experience of the laryngoscopy operators in the technique is recorded (number of previous LISA procedures). Junior operators are allowed to perform the procedure supervised by a senior operator ready to take over if required.

\section{Outcomes}

\section{Primary outcome}

The primary outcome is the need for MV from the LISA procedure onwards up to $72 \mathrm{~h}$ of life indicated according to standardized criteria:

- repeated and severe apnea (defined by the American Academy of Pediatrics Guidelines) with bradycardia and/or low oxygen saturation) [35];

- high $\mathrm{FiO}_{2}$ (according to local practices) justifying intubation for a second administration of surfactant and MV;

- respiratory complications such as pneumothorax, with an aggravation of the RDS;

- any other cause where the clinician judges MV to be necessary: The motive for the respiratory assistance is indicated in the Case Report Form (CRF). All intubations for MV is recorded.

\section{Secondary outcomes}

- The FANS score is collected before, during and after the LISA procedure in both groups (propofol and placebo).

- Ketamine administration (number of injection(s), dose for rescue (if FANS $\geq 6$ ) is also recorded.

- Per procedure events are recorded including the number of laryngoscopies needed to perform LISA, the evolution of cardiorespiratory parameters from baseline to $1,3,5,15,30,60$ and $120 \mathrm{~min}$ after the first drug injection: heart rate, respiratory rate, pulse oxymetry, blood pressure, $\mathrm{FiO}_{2}$, ventilatory mode, inspiratory and end-expiratory ventilation pressures, transcutaneous $\mathrm{pCO}_{2}$, the presence of apnoea requiring bag mask ventilation or additional nasal pressure with NIPPV and the emergency intubation for severe apnea after the drug injection before the LISA procedure.

- After the procedure: the clinician who successfully performed the LISA rates his/her performance during the laryngoscopy (especially the facility of exposure of the glottis) according to the VibyMogensen scale [33, 36]. This score based on 5 items (scored from 0 to 4), explores the facility to expose the larynx and the infant's behavior [36].
Others items related to the tolerance of the procedure such as cardiorespiratory and neurological parameters are collected at $24 \mathrm{~h}$ and $72 \mathrm{~h}$ after the intervention:

- in-hospital neonatal morbidity and mortality: pneumothorax, necrotizing enterocolitis according to Bell stage [37], proven early and late onset sepsis (respectively defined as positive CSF or blood culture before $<$ and after $>72 \mathrm{~h}$ of life), retinopathy of prematurity according to international classification [38], cystic periventricular leukomalacia or grade 3 or 4 intraventricular hemorrhage according to the Papile classification [39], surgical treatment of patent ductus arteriosus, duration of cumulated MV, duration of cumulated non-invasive ventilation, any intubation, death at 36 wGA and in-hospital mortality.

- Data at 2 years of age will be collected: Standard pediatric examination, Age and stage questionnaire (ASQ) [40] completed by the parents, Gross Motor Function Classification Score [41] in cases of motor impairment, and any visual and/or hearing disabilities detected during the first 2 years of life (Fig. 2).

\section{Consent}

Considering the emergency context of most situations, anticipated informed consent is sought from mothers, and ideally both parents, as soon as possible after the admission of the mother to the delivery room or at admission of the neonate to the NICU. It is possible to propose the study before birth if the mother is previously hospitalized in the obstetrical unit. Initially a single parent's signature is sufficient to include the newborn in the study. The recovery of the second parent's signature is sought within $48 \mathrm{~h}$ of the neonate's inclusion.

\section{Sample size}

The study has a non-inferiority objective for MV within $72 \mathrm{~h}$. The type I error is set at $5 \%$ and the power at $80 \%$. We expect a $30 \%$ rate of MV in the control group which corresponds to the percentage of intubated patients $72 \mathrm{~h}$ after the LISA procedure in the AMV study [6]. The non-inferiority margin considered as clinically acceptable is set at $10 \%$. In this setting, a total of 542 neonates will be recruited (271 per group).

\section{Recruitment and allocation}

As the total number of patients needed is 542 the study is multicentric, with the involvement of 12 motivated neonatal units in university hospitals over 3 years. Each center's estimate for recruitment was based on the usual level of activity of the NICU. 


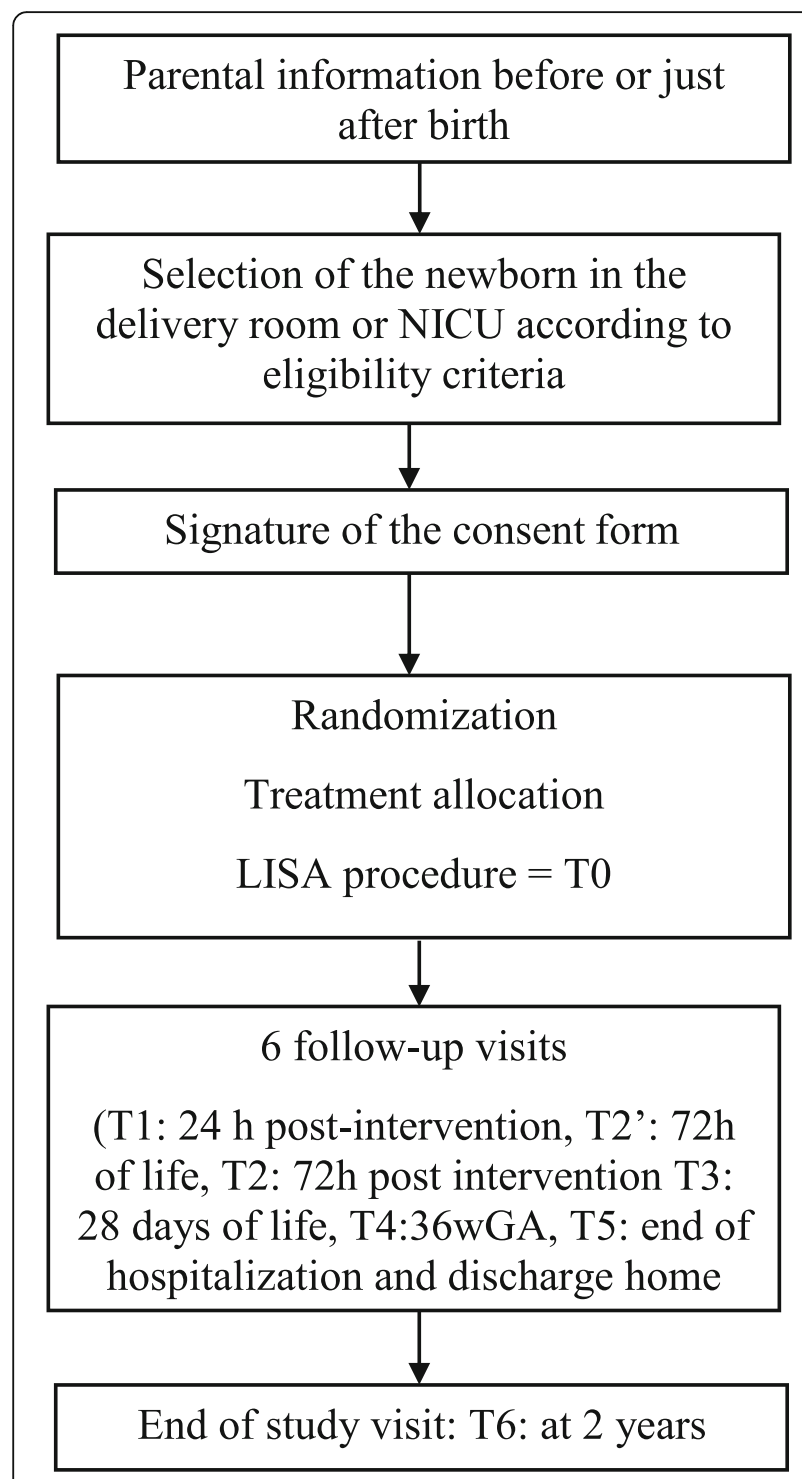

Fig. 2 Time frame

A predefined randomization list with a 1:1 ratio, obtained using a random number generator, with a random size of blocks from 2 to 6 , was established. The randomization was stratified by center and by class of GA $(<28,28-32$ wGA). Clinical staff are unaware of the allocation sequence, which is communicated only to the Grenoble hospital pharmacy.

\section{Blinding}

Drugs are delivered by the hospital pharmacy as required in sequentially numbered containers. Medialipide $^{\circ}$ is used in the control group to ensure that the 2 arms are indistinguishable. Medialipides ${ }^{\circ}$ is an emulsion of medium and long triglycerides based derived from soya oil and has the same appearance as propofol [20, 22].
Data collection, management, analysis, auditing

A learning phase involving junior and senior neonatologists has been planned in all centers to ensure the homogeneity of practice including sedation/placebo injection, monitoring during the procedure, and rescue analgesia. Training is supported by videotapes illustrating the LISA procedure and relayed by the referent neonatologist in each participating center. It also includes examples for scoring FANS. During the LISA procedure one or two observers not involved in the procedure are assigned to the collection of data (FANS, cardiorespiratory parameters etc.). Physiological parameters are extracted from the monitors to serve as source data. In each center, one clinical research assistant is in charge of entering all the data in an electronic CRF, registered with a secure interface managed by the Grenoble Alps University Hospital.

During the clinical research or after its termination, the collected data will be anonymized before being communicated to the sponsor by the investigators (or any other specialist).

A clinical research assistant mandated by the sponsor regularly visits the study centers: while the study is being set-up, one or more times during the course of the research depending on the rate of inclusions and at the end of the research. The elements to be monitored during these visits and the frequency of these visits have been defined prior to the start of the study in collaboration with the investigation team and according to an assessment of the risk level of the study. All visits are the object of a written report and a copy is forwarded to the principal investigator.

Any written or oral communication of research results must receive the prior approval of the principal/coordinating investigator and, of any committee established for the research.

\section{Harms}

All the infant's vital signs are monitored throughout and after the intervention and any significant adverse event, adverse drug reaction (hypotension, severe apnea, emergency intubation after drug injection) or unexpected adverse drug reaction (according strict definitions) are reported by the investigator to the sponsor's service in charge of safety and regardless of any causal relationship concerning the drug under investigation or the research. In case of suspected adverse reactions and if the knowledge of the treatment administered allows a specific management, unblinding can be requested by the investigator even in case of emergency. Unblinding may be done $24 / 24 \mathrm{~h}$ by the Pharmacy. A pharmacologist, and two neonatologists who are independent of the study compose the independent monitoring committee. They must assess whether there is any causal link between the 
SAE and the experimental drug or procedure. This committee meets at the request of the sponsor or principal investigator, after 50 inclusions, annually or after any intermediary analysis. They can purpose four types of action: continue following the protocol, asking for a complementary analysis, asking for a protocol amendment, stopping the study (for patient security reasons).

\section{Statistical methods}

Considering that an intention to treat analysis may not be appropriate for non-inferiority trials [42] a perprotocol analysis, including all patients who satisfactorily complied with the assigned treatment and who had no major protocol violations, will be conducted. An intention to treat analysis will be carried out subsequently to confirm the results of the per-protocol analysis. The threshold $p<0.05$ will be considered to define the significance of the statistical tests performed with Stata MP15. Blinding will be maintained during the statistical analysis.

\section{Primary outcome}

The need for MV within $72 \mathrm{~h}$ will be assessed in each group. This trial aims to show that the experimental arm with sedation is no less effective than the control arm with placebo, and we made the hypothesis that the rate of MV within $72 \mathrm{~h}$ of life will not exceed $40 \%$ with sedation, compared to $30 \%$ in the control group [6], corresponding to an equivalence margin of $10 \%$. If the upper limit of the one-sided 95\% confidence interval for the treatment difference by the experimental treatment is less than this equivalence margin, then we will retain the non-inferiority of the experimental strategy at a 5\% significance level. If non-inferiority is rejected, the rates of $\mathrm{MV}$ up to $72 \mathrm{~h}$ life will be compared using a conventional superiority test.

\section{Secondary outcomes}

The analysis of the primary outcome will be repeated by class of GA $(<28,28-31 w G A)$. Means and standard deviations (or median and 25th and 75th percentile) of FANS, cardio-respiratory parameters, number of laryngoscopies, number of apnoeas, clinician's satisfaction, neurodevelopmental outcomes at 2 years (ASQ, GMFCS, visual and hearing function), will be calculated and compared using the Student test (or Mann-Whitney test).

Numbers and frequencies of ketamine administration for rescue and emergency intubation, pneumothorax within the first $72 \mathrm{~h}, \mathrm{BPD}$ at 28 days and 36wGA, other outcomes of neonatal morbidity and in-hospital mortality will be calculated, and compared using the Chi2 test (or Fischer exact test).

\section{Interim analysis}

An interim analysis of the primary endpoint will be performed once half the planned number of newborns have been included. This interim analysis will aim to confirm patient safety and help decide on the continuation or discontinuation of the study (stopping for toxicity or stop for futility). To maintain an overall level of $5 \%$ in the final analysis, the interim analysis will be performed with a threshold of $0.1 \%$. The results of the interim analysis will be discussed by the steering committee and safety committee who may propose amendments to the statistical analysis plan.

\section{Missing data}

Considering the design and that endpoints are mostly recorded during hospitalization, a high rate of missing data is unlikely. Nevertheless, in case of rates between 5 and $20 \%$, missing data will be replaced. The replacement of missing data will either be according to an analysis strategy for the worst case scenario, or by multiple imputation. If multiple imputation is used, 5 imputations will be made, using a logistic regression model taking into account the main factors related to the outcome.

\section{Research ethics approval}

The study n²018-002876-41 (EudraCT) was approved by the French research ethics committee on March 3, 2019 and by the French drug administration, on October 5 , 2018. If there is a need for protocol amendments, the principal investigator has to inform the different investigators, trial participants, the promotor and the amendments should be presented to the French research ethic committee for approval.

\section{Discussion}

In Europe the widespread use of the LISA technique and the known deleterious effects of laryngoscopy in unsedated neonates prompted researchers to evaluate the feasibility of premedication for the LISA procedure. In the study by Heiring et al., of 73 North European NICUs performing the LISA procedure $78 \%$ declared using medication with a large variety of drugs [30]. Currently the most studied drug is propofol $[11,12,20]$ with promising preliminary results from a single center, unmasked, randomized controlled trial versus no premedication [20]. Moreover, we did not choose fentanyl as a rescue treatment due to the major risk of chest wall rigidity and thus intubation $[14,43]$.

Our protocol has two limitations: the open label treatment with ketamine in both groups and the multiple possible indications for intubation. Concerning the first limitation, a "no sedation" control group for a painful procedure (LISA) seems to be unethical. Therefore we planned rescue treatment using ketamine as suggested 
by Berde et al. for pediatric analgesic trials [34]. This design is not perfect to evaluate a medication, but it seems to be best for the included neonate. Ketamine rescue and the number of doses required will be an interesting factor to compare between the two groups. It may reflect the potential pain management across the both group.

Regarding the second limitation, we considered that the use of mechanical ventilation after a LISA procedure should be similar to real-life practice. Although indications for MV were not strictly standardized, the results obtained will have reinforced generalisability. This approach was also adopted in the initial AMV trial [6] and has been retained in the present study. If our hypothesis of non-inferiority and safety is verified, it provides a strong argument for performing LISA with propofol sedation. This could have an impact on the generalization of LISA, which may have real benefits for infants with bronchopulmonary dysplasia.

This trial will be the first multicenter, double blind, randomised, placebo controlled trial evaluating premedication before LISA with an expected number of participants of 542. In addition it will examine the neurodevelopmental outcome at 2 years of age of preterm infants who received a few doses of propofol or ketamine shortly after birth [22, 44].

Our study should provide robust data on premedication use before a routine LISA procedure for clinicians studying tolerance, efficacy and adverse events. Furthermore, it may contribute to improving the comfort and pain management of neonates during the LISA procedure.

\section{Abbreviations}

BDP: Bronchopulmonary dysplasia; CRF: Case report form; FANS: Face acute neonatal scale; LISA: Less invasive surfactant administration; MV: Mechanical ventilation; NICU: Neonatal intensive care unit; NIPPV: Non-invasive positive pressure ventilation; RDS: Respiratory distress syndrome; wGA: weeks of gestational age
}

\section{Acknowledgments}

We thank members of the PROLISA Study group: Alain Beuchee (Rennes), Laura Bourgoin (Mareseille), Aurélie Desenfants (Nîmes), Amélie Durandy (Poissy), Cyril Flamant (Nantes), Géraldine Gascoin (Angers), Ghida Ghostine (Amiens), Johanna Parra (Chambery), Laure Ponthier (Limoges) and JeanMichel Roué (Brest). We also thank all the participating NICUs for their collaboration and Alison Foote (Grenoble Alps University Hospital) for editing the manuscript.

\section{Authors' contributions}

$T D$ is the chief clinician and principal investigator; $A E, X D, M C$ and the ProLISA study group are co-investigators. TD with $A E, X D, M C$ designed and set-up this study. All authors read, made suggestions for changes and approved the final version of the manuscript.

\section{Authors' information}

TD (MD, PhD) is the head of neonatal intensive care unit at Grenoble Alps University Hospital. He studied pain in neonates and his current research interests include the LISA procedure and neonatal neurology (hypoxicischemic encephalopathy). AE (MD, PhD) is an epidemiologist at Grenoble Alps University Hospital specializing in neonatal epidemiology. She works in neonatal neurological studies and antenatal projects. XD ((MD, PhD) works on analgesia during intubation and the LISA procedure, he currently works at Creteil Hospital. MC (MD) is PhD student whose the topics of research are the LISA procedure, pain and neonatal neurology especially the epidemiology of intraventricular hemorrhage. She currently works at Grenoble Alps University Hospital.

\section{Funding}

This research involving humans is funded by the National Hospital Clinical Research Program (PHRC-DGOS, project number PHRCN-1701-76), of the French Ministry of Health. The call for projects takes place every year. The project was therefore the subject of a peer-review with at least 2 French and one foreign expertise to be selected. Sponsor is Grenoble Alps University Hospital (CHU Grenoble Alps), Pavillon Dauphiné, CS 10217, 38043 Grenoble Cedex 09 .

\section{Availability of data and materials}

The data recorded during this research are subject to computer processing by the clinical research center, Grenoble Alps University Hospital in accordance with Law No. 78-17 of 6 January 1978 on computers, files and freedoms amended by Law 2004-801 of August 6, 2004.

\section{Ethics approval and consent to participate}

The study n²018-002876-41 (EudraCT) was approved by the French research ethics committee on March 3, 2019 and by the French drug administration, on October 5, 2018. This one covers all study sites during the study. Considering the emergency context of most situations, anticipated written informed consent is sought from mothers, and ideally both parents, as soon as possible after the admission of the mother to the delivery room or at admission of the neonate to the NICU.

\section{Consent for publication}

All authors have given the consent for publication.

\section{Competing interests}

The authors declare that they have no competing interests.

\section{Author details}

1UMR 5525 ThEMAS, CNRS, TIMC-IMAG, Grenoble Alps University, Grenoble, France. ${ }^{2}$ Neonatal Intensive Care Unit, Grenoble Alps University Hospital, Grenoble, France. ${ }^{3}$ Neonatal Intensive Care Unit, Centre Hospitalier Intercommunal de Créteil, Créteil, France. ${ }^{4}$ Université Paris Est, IMRB- GRC GEMINI, Créteil, France. ${ }^{5}$ Inserm, U1153, Obstetrical, Perinatal and Pediatric Epidemiology Team, Epidemiology and Biostatistics Sorbonne, Paris Descartes University, Paris, France.

Received: 14 April 2020 Accepted: 29 April 2020

Published online: 08 May 2020

\section{References}

1. Laughon MM, Langer JC, Bose CL, Smith PB, Ambalavanan N, Kennedy KA, et al. Prediction of Bronchopulmonary dysplasia by postnatal age in extremely premature infants. Am J Respir Crit Care Med. 2011;183:1715-22.

2. Balany J, Bhandari V. Understanding the impact of infection, inflammation, and their persistence in the pathogenesis of Bronchopulmonary dysplasia. Front Med. 2015:2:90.

3. Jensen EA, DeMauro SB, Kornhauser M, Aghai ZH, Greenspan JS, Dysart KC. Effects of multiple ventilation courses and duration of mechanical ventilation on respiratory outcomes in extremely low-birth-weight infants. JAMA Pediatr. 2015:169:1011-7.

4. Fischer HS, Bührer C. Avoiding endotracheal ventilation to prevent Bronchopulmonary dysplasia: a meta-analysis. Pediatrics. 2013;132:e1351-60.

5. Sweet DG, Carnielli V, Greisen G, Hallman M, Ozek E, te Pas A, et al. European consensus guidelines on the Management of Respiratory Distress Syndrome - 2019 update. Neonatology. 2019;115:432-50.

6. Göpel W, Kribs A, Ziegler A, Laux R, Hoehn T, Wieg C, et al. Avoidance of mechanical ventilation by surfactant treatment of spontaneously breathing preterm infants (AMV): an open-label, randomised, controlled trial. Lancet. 2011:378:1627-34

7. Kanmaz HG, Erdeve O, Canpolat FE, Mutlu B, Dilmen U. Surfactant administration via thin catheter during spontaneous breathing: randomized controlled trial. Pediatrics. 2013;131:e502-9. 
8. Mohammadizadeh M, Ardestani AG, Sadeghnia AR. Early administration of surfactant via a thin intratracheal catheter in preterm infants with respiratory distress syndrome: feasibility and outcome. J Res Pharm Pract. 2015:4:31-6.

9. Bao Y, Zhang G, Wu M, Ma L, Zhu J. A pilot study of less invasive surfactant administration in very preterm infants in a Chinese tertiary center. BMC Pediatr. 2015;15. https://doi.org/10.1186/s12887-015-0342-7.

10. Kribs A, Roll C, Göpel W, Wieg C, Groneck P, Laux R, et al. Nonintubated surfactant application vs conventional therapy in extremely preterm infants: a randomized clinical trial. JAMA Pediatr. 2015;169:723.

11. Dekker J, Lopriore E, Rijken M, Rijntjes-Jacobs E, Smits-Wintjens V, te Pas A. Sedation during minimal invasive surfactant therapy in preterm infants. Neonatology. 2016;109:308-13.

12. Descamps CS, Chevallier M, Ego A, Pin I, Epiard C, Debillon T. Propofol for sedation during less invasive surfactant administration in preterm infants. Arch Dis Child Fetal Neonatal Ed. 2017;102:F465.

13. Bourgoin L, Caeymaex L, Decobert F, Jung C, Danan C, Durrmeyer $X$. Administering atropine and ketamine before less invasive surfactant administration resulted in low pain scores in a prospective study of premature neonates. Acta Paediatr. 2018;107:1184-90.

14. Olivier F, Nadeau S, Bélanger S, Julien A-S, Massé E, Ali N, et al. Efficacy of minimally invasive surfactant therapy in moderate and late preterm infants: a multicentre randomized control trial. Paediatr Child Health. 2017;22:120-4.

15. Dargaville PA, Kamlin COF, De Paoli AG, Carlin JB, Orsini F, Soll RF, et al. The OPTIMIST-A trial: evaluation of minimally-invasive surfactant therapy in preterm infants 25-28 weeks gestation. BMC Pediatr. 2014;14:213.

16. Aldana-Aguirre JC, Pinto $M$, Featherstone RM, Kumar $M$. Less invasive surfactant administration versus intubation for surfactant delivery in preterm infants with respiratory distress syndrome: a systematic review and metaanalysis. Arch Dis Child Fetal Neonatal Ed. 2017;102:F17-23.

17. Rigo V, Lefebvre C, Broux I. Surfactant instillation in spontaneously breathing preterm infants: a systematic review and meta-analysis. Eur J Pediatr. 2016; 175:1933-42.

18. Klotz D, Porcaro U, Fleck T, Fuchs H. European perspective on less invasive surfactant administration-a survey. Eur J Pediatr. 2017;176:147-54.

19. Tauzin $M$, Durrmeyer $X$. Managing neonatal pain in the era of non-invasive respiratory support. Semin Fetal Neonatal Med. 2019;24:101004.

20. Dekker J, Lopriore E, van Zanten HA, Tan RNGB, Hooper SB, Te Pas AB. Sedation during minimal invasive surfactant therapy: a randomised controlled trial. Arch Dis Child Fetal Neonatal Ed 2018;104:F378-F383.

21. Chidambaran V, Costandi A, D'Mello A. Propofol: a review of its role in pediatric anesthesia and sedation. CNS Drugs. 2015;29:543-63.

22. Durrmeyer X, Breinig S, Claris O, Tourneux P, Alexandre C, Saliba E, et al. Effect of atropine with Propofol vs atropine with Atracurium and Sufentanil on oxygen desaturation in neonates requiring nonemergency intubation: a randomized clinical trial. JAMA. 2018;319:1790-801

23. Vedrenne-Cloquet $M$, Breinig S, Dechartres A, Jung C, Renolleau S, Marchand-Martin L, et al. Cerebral oxygenation during neonatal intubationancillary study of the Prettineo-study. Front Pediatr. 2019;7:40.

24. Bhutta AT. Ketamine: a controversial drug for neonates. Semin Perinatol. 2007:31:303-8.

25. Barois J, Tourneux P. Ketamine and atropine decrease pain for preterm newborn tracheal intubation in the delivery room: an observational pilot study. Acta Paediatr. 2013;102:e534-8.

26. Kumar P, Denson SE, Mancuso TJ. Committee on fetus and newborn, section on anesthesiology and pain medicine. Premedication for nonemergency endotracheal intubation in the neonate. Pediatrics. 2010;125: 608-15.

27. Millar C, Bissonnette B. Awake intubation increases intracranial pressure without affecting cerebral blood flow velocity in infants. Can J Anaesth. 1994:41:281-7.

28. Marshall TA, Deeder R, Pai S, Berkowitz GP, Austin TL. Physiologic changes associated with endotracheal intubation in preterm infants. Crit Care Med. 1984;12:501-3

29. Barrington K. Premedication for endotracheal intubation in the newborn infant. Paediatr Child Health. 2011;16:159-71.

30. Heiring C, Jonsson B, Andersson S, Björklund L. Survey shows large differences between the Nordic countries in the use of less invasive surfactant administration. Acta Paediatr. 2017;106:382-6.

31. Stevens TP, Harrington EW, Blennow M, Soll RF. Early surfactant administration with brief ventilation vs. selective surfactant and continued mechanical ventilation for preterm infants with or at risk for respiratory distress syndrome. Cochrane Database Syst Rev. 2007:4:CD003063.

32. Henderson-Smart DJ, Steer P. Methylxanthine treatment for apnea in preterm infants. Cochrane Database Syst Rev. 2001;3:CD000140.

33. de Kort EHM, Andriessen P, Reiss IKH, van Dijk M, Simons SHP. Evaluation of an intubation readiness score to assess neonatal sedation before intubation. Neonatology. 2019;115:43-8.

34. Berde CB, Walco GA, Krane EJ, Anand KJS, Aranda JV, Craig KD, et al. Pediatric analgesic clinical trial designs, measures, and extrapolation: report of an FDA scientific workshop. Pediatrics. 2012;129:354-64.

35. Eichenwald EC, Newborn C on FA. Apnea of Prematurity. Pediatrics. 2016; 137:e20153757.

36. Viby-Mogensen J, Engbæk J, Eriksson LI, Gramstad L, Jensen E, Jensen FS, et al. Good clinical research practice (GCRP) in pharmacodynamic studies of neuromuscular blocking agents. Acta Anaesthesiol Scand. 1996:40:59-74

37. Bell MJ, Ternberg JL, Feigin RD, Keating JP, Marshall R, Barton L, et al. Neonatal necrotizing enterocolitis. Therapeutic decisions based upon clinical staging. Ann Surg. 1978;187:1-7.

38. International Committee for the Classification of Retinopathy of Prematurity. The international classification of retinopathy of prematurity revisited. Arch Ophthalmol Chic. 2005;123:991-9.

39. Papile LA, Burstein J, Burstein R, Koffler H. Incidence and evolution of subependymal and intraventricular hemorrhage: a study of infants with birth weights less than 1,500 gm. J Pediatr. 1978;92:529-34.

40. Squires J, Twombly E, Bricker D, Potter L. ASQ-3 User's Guide. Brookes Publishing Co. Inc. Baltimore (USA). Paul H; 2009.

41. Palisano R, Rosenbaum P, Walter S, Russell D, Wood E, Galuppi B. Development and reliability of a system to classify gross motor function in children with cerebral palsy. Dev Med Child Neurol. 1997;39:214-23.

42. Schumi J, Wittes JT. Through the looking glass: understanding noninferiority. Trials. 2011;12:106.

43. Fahnenstich H, Steffan J, Kau N, Bartmann P. Fentanyl-induced chest wall rigidity and laryngospasm in preterm and term infants. Crit Care Med. 2000; 28:836-9.

44. Elalouf C, Le Moing A-G, Fontaine C, Leke A, Kongolo G, Gondry J, et al Prospective follow-up of a cohort of preterm infants $<33$ WG receiving ketamine for tracheal intubation in the delivery room: neurological outcome at 1 and 2 years. Arch Pediatr. 2018;25:295-300.

\section{Publisher's Note}

Springer Nature remains neutral with regard to jurisdictional claims in published maps and institutional affiliations.

Ready to submit your research? Choose BMC and benefit from:

- fast, convenient online submission

- thorough peer review by experienced researchers in your field

- rapid publication on acceptance

- support for research data, including large and complex data types

- gold Open Access which fosters wider collaboration and increased citations

- maximum visibility for your research: over $100 \mathrm{M}$ website views per year

At $\mathrm{BMC}$, research is always in progress.

Learn more biomedcentral.com/submissions 